\title{
Structuring Contract-for-Difference Instruments for Hedging Electricity Price Risks on a Blockchain-based Marketplace
}

\author{
Olakunle Alao, Student Member, IEEE and Paul Cuffe, Member, IEEE
}

\begin{abstract}
The volatile nature of day-ahead electricity markets means that participants often resort to some form of derivative hedging instrument. One such derivative instrument is a Contractfor-Difference (CfD), specifically available to renewable generators in some jurisdictions to enable them to hedge against their price risk. CfD is a bilateral arrangement between a generator selling into, and an offtaker buying out of, a centrally cleared pool market for electricity. In this arrangement, the generator subsidizes the offtaker when the spot price is high; whereas, the offtaker subsidizes the generator when the spot price is low. This establishes a synthetic bilateral electricity transaction, operating in parallel to the pool market. Embracing CfD to hedge against price risk presents new risks such as counterparty credit, margining, third-party, and legal risks. They also incur high costs and possess underlying process risks. Decentralized Finance - an overarching term representing financial services built on top of a public blockchain - seems to present particularly compelling opportunities in electricity derivatives for these reasons. Therefore, we propose a novel Decentralized Finance instrument: a blockchain-based marketplace governed by a smart contract to act as a mediator between stakeholders mutually enrolled in bilateral CfD arrangements. The employed smart contract structure autonomously and irrefutably enforces the terms of the CfD, underpinned by a novel collateralization and settlement mechanism. This novel approach mitigates the hedging-related and underlying process risks of traditional CfD instruments.
\end{abstract}

Key words-Decentralized Finance, Electricity derivatives, Blockchain, Smart contracts and Renewable electricity

\section{INTRODUCTION}

$\mathbf{T}$ HE diminishing level of fossil fuels, greenhouse gas emissions that accompany their exploitation, as well as increasing global electricity demand, is triggering the need for accelerated renewable electricity development [1].

In day-ahead electricity markets, generators primarily participate in the pool market, as in Fig. 1. Here, they deliver power physically into the grid. In return, they secure revenue proportional to the volume of electricity they supply and the uniform clearing price of the pool market for that trading period [1]. The unique physical characteristics of electricity

This publication has been funded by the Sustainable Energy Authority of Ireland under the SEAI Research, Development \& Demonstration Funding Program 2018, grant number 18/RDD/373.

O. Alao (olakunle.alao@ucdconnect.ie) and P. Cuffe are with the School of Electrical and Electronic Engineering, University College Dublin.

Interests disclosure: The authors hold cryptographic assets.

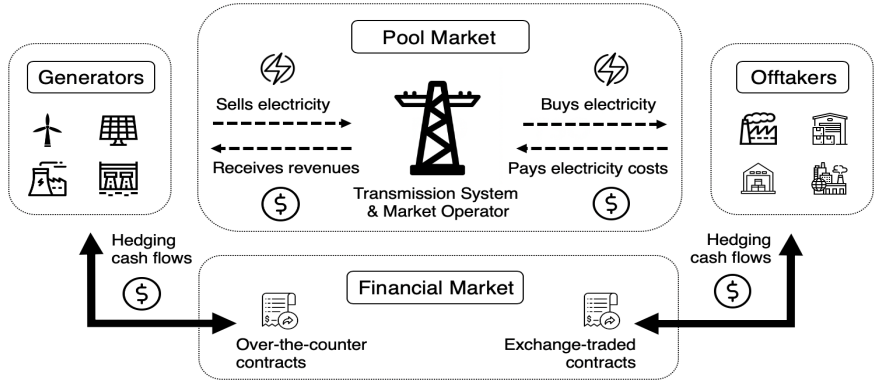

Fig. 1. Basic structure of a notional day-ahead electricity market.

generation and distribution, reflected in day-ahead electricity markets, makes these spot prices quite volatile [2]. Therefore, generators could be exposed to the risk of sustained low prices, resulting in depressed revenues. Renewable generators, in particular, are also susceptible to volume risk [3], as the output of wind or PV plants, for instance, are variable; and thus liable to underperform expectations for days, weeks, months or even years [3]. These inherent risks to the revenue stream of prospective renewable electricity projects, amongst others, could make mobilizing finance from traditional risk-averse sources quite challenging [4].

For this reason, many government subsidy schemes aim to derisk renewable electricity development [5]. Complementary to these, a range of specific financial derivative instruments have emerged to stabilize revenue streams for renewable generators [6], [7]. Overall, generators (renewable and conventional) usually resort to some form of these derivative instruments in the financial market, parallel to the centrally cleared pool market for electricity, as in Fig. 1 [7]. These derivatives are typically traded over-the-counter or on organized exchanges. Classical forward contracts on electricity prices are bilaterally traded over-thecounter; hence, their terms are negotiable. Conversely, futures on electricity prices are traded on organized exchanges with standardized terms [2], [7]. In practice, generators typically hold a balanced portfolio of these derivatives, as each instrument possesses unique weaknesses and strengths [2], [7].

\section{A. Contract-for-Difference}

A specific financial derivative instrument that is available in, for example, the UK and Australian day-ahead electricity 
markets to stabilize revenue streams for renewable generators, is the Contract-for-Difference (CfD) [5]. CfD instruments are forward electricity derivatives that achieve revenue stability for renewable generators and offtakers by hedging against their spot price risk exposure. They are undertaken between a renewable generator and a counterparty offtaker who buys out of the same pool that the generator sells into [7]. As high spot prices are good for the generator but bad for the offtaker, and low spot prices are the opposite, it is mutually beneficial for these parties to cooperate in hedging each other's exposure to these volatile spot prices. Importantly, a CfD does not hedge against volume risk; hence, renewable generators maintain the volume risk of over- or under-realizing their predicted delivery volume.

In a $\mathrm{CfD}$, contracting parties receive or pay the difference between the trading period spot price and pre-agreed strike price per unit of electricity over a specific agreement duration [1]. If the strike price is greater than the spot price, the generator receives a payoff from the offtaker - the difference between the strike price and spot price per unit of electricity (Mwh). Otherwise, the generator returns a payoff to the offtaker - the difference between the spot price and strike price per unit of electricity (Mwh) [5]. In simple terms, CfD could be seen as a medium-term or long-term Virtual Power Purchase Agreement between bilateral parties that protect them from the volatile spot price risk of the day-ahead electricity market as in Fig 2 [8]. CfDs are Virtual Power Purchase Agreements because they are financial instruments and hence do not involve the delivery or evacuation of physical power [5].

Traditional CfD instruments are traded over-the-counter like other bilateral contracts [9]. Thus, they are liable to counterparty credit risk, resulting from the possibility that a contracting party can incur a financial loss because of a default on settlement payment by its bilateral counterparty [7]. They are exposed to high margining risks because of the non-frequent settlement times of the central intermediary that usually clear and settle payments due to contracting parties [10]. The non-frequent settlement times result in accumulated settlement payments. This imposes the need for contracting parties to maintain high margins to reflect the quantum of cash flows to be hedged and thus introduces the risk of high margin payments [1].

Further, traditional CfDs are susceptible to third-party risks, due to the probability that the intermediary (known as a broker) underpinning transactions can become fraudulent or insolvent [7]; and legal risks arising from possible contractual disputes between contracting parties [2]. Traditional CfDs also incur high direct (transaction-related) and indirect costs [9]; and possess underlying process risks, the probability that a contracting party can suffer a financial loss because the process that supports the CfD arrangement lacks operational efficiency and reliability [9]. In the context of traditional CfDs, process risks result from the error-susceptible manual moving parts that slows non-transaction and transaction-related activities of the contract.

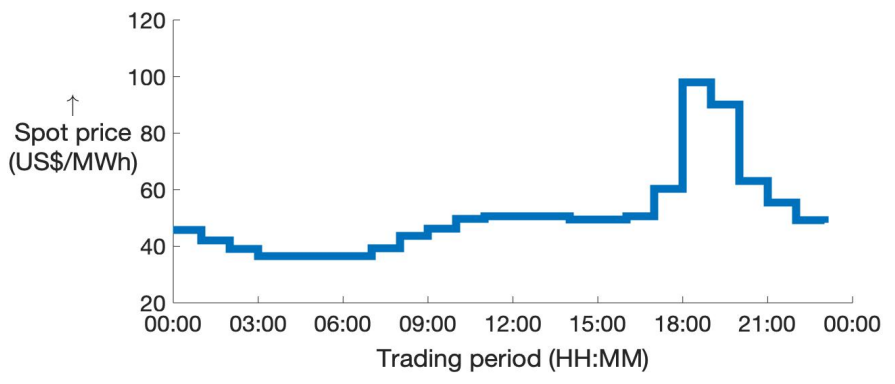

Fig. 2. Actual trading day spot prices of a day-ahead electricity market in Europe denominated in us\$ [8].

\section{B. Decentralized Finance}

Decentralized Finance (DeFi) can be defined as financial services running on the blockchain [11] - an immutable and decentralized digital ledger that enables transactions between parties to be recorded in a secure and permanent manner [12], [13]. The operation of DeFi instruments on the blockchain is governed by smart contracts. Smart contracts are special computer scripts (or chaincode) that execute natively within a blockchain in a way that cannot be impeded or interfered with [14]. For instance, a smart contract can autonomously hold and manage cryptographic assets based on predefined conditions. When the appropriate conditions are met, the smart contract can autonomously disburse funds to a particular counterparty or token-holder [15].

DeFi principles are quite new in electricity derivatives. The earliest work on DeFi electricity derivative products was undertaken in [16]. Here, the main regulatory issues around the implementation of blockchain-based electricity wholesale trading for over-the-counter transactions were investigated. Since then, not much work has been achieved in the blockchain electricity derivatives space. The present authors [1] proposed a more applied form of the earlier work in [16], where they sketched out the basic framework for a blockchain CfD derivative instrument in the electricity industry. Still, this project was exploratory and intended to serve as an impetus towards the development of advanced and robust blockchain electricity derivatives, as our work here.

The financial industry has seen more substantial proposals and applications of DeFi derivatives [17], [18], [19]. Here, DeFi is proving to reduce some hedging-related and underlying risks of traditional financial derivatives. For example, in [17], DeFi derivative products were proposed to hedge systemic risk in forwards over-the-counter contracts. The exposure to systemic risk was reported to arise from the non-transparent reporting of over-the-counter transactions to trade repositories, tasked with monitoring these transactions [17]. In [18], [19], a derivative market was implemented on a public blockchain to reduce counterparty credit risk. The results here also indicate mitigation of third-party risks since the role of intermediaries who introduce these risks are made redundant by the self-executing blockchain smart contracts. The trading 
of Bitcoin futures on the Chicago Board Options Exchange and the Chicago Mercantile Exchange represents an applied implementation of DeFi derivative products [20].

DeFi derivative instruments could potentially be a good fit for the electricity industry for the following reasons. First, the presence of several non-transaction and transaction-related manual moving parts in the electricity industry's financial market [2] presents compelling opportunities for blockchain smart contracts proven to automate manual processes inherent in traditional financial instruments [13], [21]. Most importantly, the mature, organized and transparent setup of wholesale electricity markets [6], [16] could allow DeFi derivative instruments to easily synchronize with these markets. To put this into context, we consider the implementation of a smart contract in an opaquely-structured industry like agriculture. Here, certain farmers might want to secure a derivative to hedge the volume risk of their harvest; however, how can a smart contract verify that some of these potatoes haven't rotted before the delivery day? Conversely, electricity is a pure fungible commodity traded in an open and transparentlystructured market that clears and settles at a known frequency [7]. This well-coordinated setup of electricity markets could allow for seamless integration of blockchain smart contracts alongside them, with little or no disruption to the existing physical trading structure.

\section{Novel contribution}

The novel contribution of the present work is a functional description of a blockchain smart contract that operates as a mediator between stakeholders mutually enrolled in bilateral $\mathrm{CfD}$ arrangements. This CfD instrument is structured as smart contract chaincode, underpinned by a novel autonomous collateralization and settlement mechanism. This smart contract framework, named Blockchain Electricity Derivative (BED), attends to the following issues, where the first two issues concern traditional CfD instruments and the last issue pertains to smart contract development:

1) How can we mitigate hedging-related risks introduced by traditional CfD instruments?

2) How can we reduce costs and the underlying process risks of traditional CfD instruments?

3) How can we mitigate some of the new exotic risks associated with DeFi applications?

We attend to the first and second issues by leveraging a blockchain smart contract platform. To mitigate hedging-related risks introduced by traditional CfD instruments, we propose the incorporation of a novel autonomous collateralization and settlement mechanism into the smart contract. The autonomous collateralization and settlement mechanisms underpin the smart governance system through interconnected financial incentives that compel off-chain actions of contracting parties that do not have to trust or know each other. The design and implementation of these mechanisms are detailed in Section II.
The decentralized and immutable attribute of blockchain [22], [23] along with the process automation introduced by the smart contract [14], [15], enables cost-reduction and addresses the underlying process risks of traditional CfD instruments [9]. The two components of process risk are the risks of lack of process efficiency and process reliability [9]. Process automation attends to the efficiency component of process risk by reducing the typical manual moving parts inherent in traditional legalistic CfD instruments. The consequence of process efficiency is the elimination of some cost components of traditional CfD instruments. These costs are usually associated with clearing \& settlement, enforcement \& compliance, reporting, adjudication processes, etc. [10]. The presence of many human intermediaries in traditional CfD instruments also introduces the risk of lack of process reliability [9]. This risk is addressed in our approach because smart contracts are persisting and function based on only the predefined conditions embedded in them [14].

Importantly, the smart contract is designed so that it is neither a forwards nor a futures contract. Instead, it fuses the characteristics of both contracts - it maintains the bilateral terms negotiability of traditional CfD instruments traded overthe-counter, albeit within a programmable smart contract; while also exploiting the economies of scale and liquidity provided by the market-based futures contract. The hybrid nature of BED should make it highly liquid and further its cost-effectiveness.

By addressing the first and second problem statements using a DeFi application, new risks are introduced into BED. These risks include volatility risk of the native currency of the blockchain [24]; security risks as a result of poorly tested smart contracts or new attack vectors and dependencies on other DeFi applications [25], [26], [27]; account management-related risks due to user errors and design risks due to faultily designed smart contracts [19]. We mitigate some of these risks, firstly, by integrating a stablecoin cryptocurrency to our smart contract to isolate our DeFi instrument against cryptocurrency price volatility [28]. Further, we mitigate design risk by ensuring that the core features of traditional CfDs are maintained in BED, as will be seen in Section II.

The rest of this paper is structured as follows. Section II presents the design methodology of the BED financial instrument. Section III presents the test platform used to showcase the workings and value of BED. Section IV demonstrates the expected functioning of BED. Section V concludes this paper.

\section{Methodology}

Traditional CfD instruments, while hedging generators and offtakers against price risk, present new risks to them: counterparty credit, margining, third-party, and legal risks. They also incur high costs and entail underlying process risks. In this section, we describe the structure of our DeFi CfD instrument that addresses these issues, under the assumption that the pool market as in Fig. 1 is maintained. Our proposed financial instrument (see Fig. 3) resides in the financial market, 


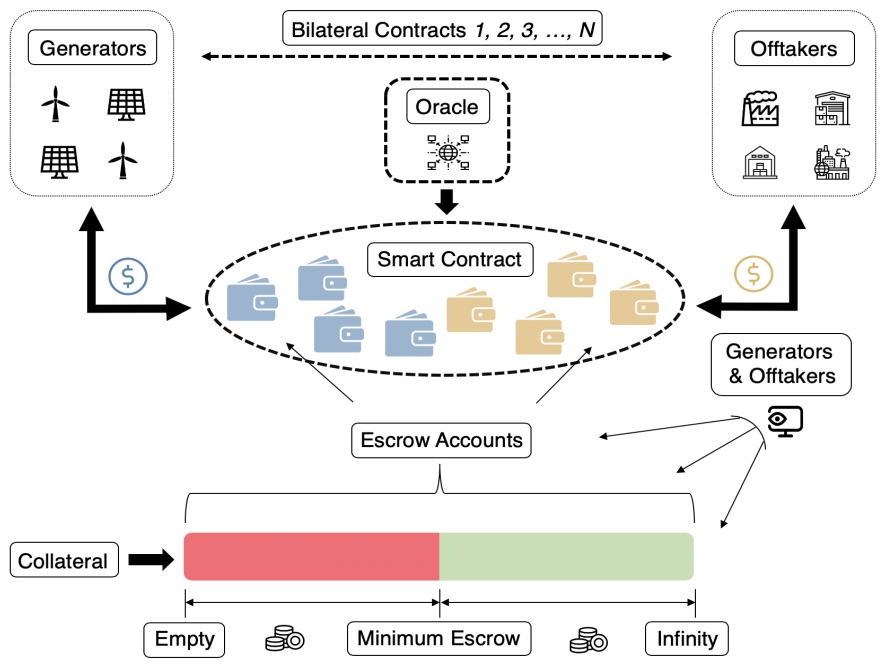

Fig. 3. Schematic of the BED financial instrument which enforces an enduring on-chain bilateral CfD arrangement between numerous parties in parallel to the pool market for electricity.

which operates alongside the pool market and is available to market participants along with other electricity derivatives.

Developing DeFi financial instruments can be challenging since the blockchain smart contract that underpins their operation must retain the equivalent legal, technical, and commercial functionalities of the traditional contract that they replace [19]. This illustrates the importance of the business logic, the core rules of the smart contract, where all the asset-handling functionalities and incentive mechanisms that encourage rational behavior are housed [1]. Central to the embodiment of the smart contract's business logic for our proposed DeFi financial instrument is a novel autonomous collateralization and settlement mechanism. Here, we will show how these mechanisms within the smart contract incentivize contracting parties that do not have to trust or know each other to maintain an enduring $\mathrm{CfD}$ hedging arrangement.

Traditional CfD instruments can be exposed to high margining risks because of the non-frequent settlement times of the bureaucratic clearing and settlement intermediaries that manage CfD payoffs of contracting parties [10]. To remove the need for a clearing and settlement intermediary, we propose a novel autonomous settlement mechanism. This mechanism ensures that at every trading period, the on-chain escrow account of each contracting party is immutably updated with their CfD payoff by the smart contract following receipt of the spot price feed. This makes settlement more rapid and dependable and mitigates margining risks. Moreover, the settlement calculations are automated in a way that is immune to interference from any party.

Counterparty credit risk is one of the main risks posed to contracting parties in a derivative transaction: what if a party in a particular bilateral arrangement fails or refuses to crosssubsidize its counterparty? In traditional CfDs, the clearing and settlement human intermediary is tasked with holding collaterals of parties to reduce their credit risk exposure during settlement. And in the event of a default, the counterparty can seize these collaterals [29]. The challenge of designing a blockchain-based CfD arrangement is, therefore, in determining how non-trusting or possibly anonymous contracting parties can be compelled to fulfill their bilateral cross-subsidizing obligations without the third-party risks introduced by human intervention.

To this end, we propose a novel autonomous collateralization mechanism that mitigates counterparty credit risk on-chain and in a fully autonomous way. Our collateralization mechanism leverages financial incentives to encourage contracting parties to secure and maintain collateral in an escrow account within the smart contract until the end of their agreement duration. Importantly, this escrow account is the same account where the CfD payoff settlement due to a contracting party is credited and debited from. Hence, contracting parties are inherently incentivized to monitor the escrow accounts of their bilateral counterparty and invoke a default call $^{1}$ if they fall below the minimum escrow requirement.

To hedge the cryptocurrency volatility risk inherent in DeFi applications, we integrate a stablecoin into our smart contract [28]. A stablecoin is a cryptocurrency that is directly pegged to a relatively-stable fiat currency. Again, the stablecoin service introduces an attack vector and thus exposes the smart contract to security risks. Stablecoin services are, however, seen as a mature technology supporting a proliferation of DeFi applications, such as in [30]. The rest of this section presents a more in-depth design of the proposed illustrative business logic.

\section{A. Autonomous settlement mechanism imposed on parties enrolled in the BED smart contract}

Traditional CfD instruments can be exposed to high margining risk, a financial risk that potential cash flows are lower due to the payment of maintenance margins [9]. The high margining risks prevalent in traditional CfD instruments are a result of the non-frequent settlement times of the bureaucratic clearing and settlement intermediaries that enforce CfD payoffs of contracting parties [1], [5]. To remove the need for a clearing and settlement intermediary, we propose a novel autonomous settlement mechanism. The autonomous settlement mechanism is directly enforced by a function within the smart contract itself. At every trading period, this function calculates the CfD payoff due to each contracting party based on a spot price feed into the smart contract, as shown in Fig. 4. Thereafter, it autonomously updates the escrow account of the respective contracting parties based on their calculated CfD payoffs.

The CfD payoff $p$ for a generator $i$ at every trading period $\forall t \in T$ is shown in (1) [1]. $V$ is the contracted volume, $s_{t}$ is the spot price, and $K$ is the strike price. From (1), if the strike

${ }^{1}$ This is a function within the BED smart contract that can be called by a contracting party when its bilateral counterparty defaults on its minimum escrow requirement. 


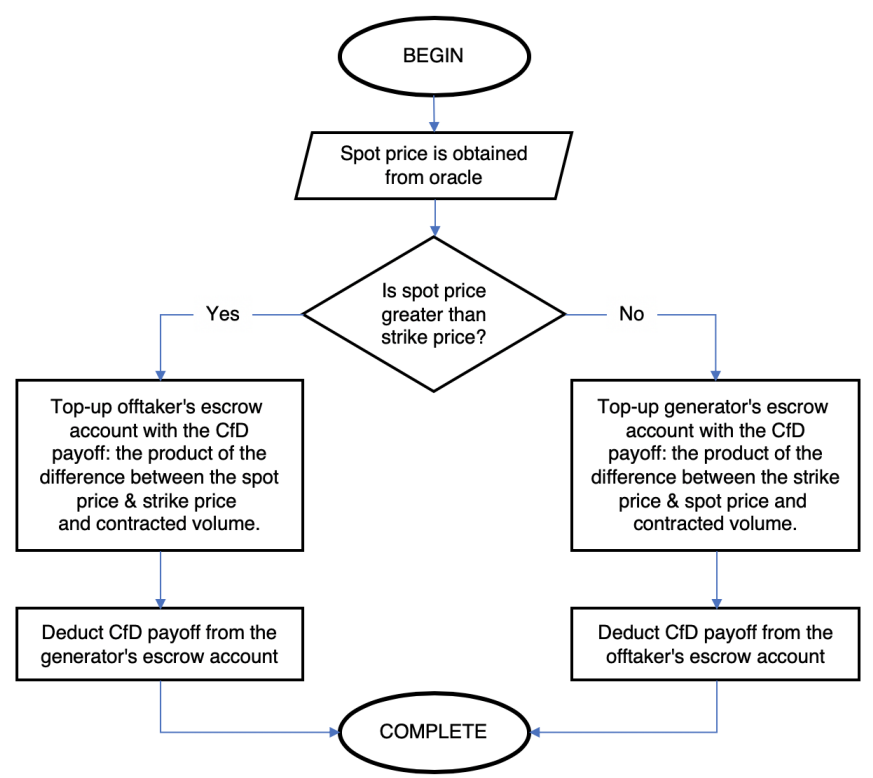

Fig. 4. Flow chart of the procedure for settlement that is irrevocably enforced by the smart contract at every trading period.

price is greater than the spot price, the generator $i$ receives a payoff from the offtaker $j$, which is the product of the difference between the strike price $\&$ spot price and the contracted volume [5]. Otherwise, the generator $i$ returns to the offtaker $j$ a payoff as in (2), which is the product of the difference between the spot price \& strike price and contracted volume [5].

$$
\begin{aligned}
& p_{j \rightarrow i}=\left(V \times \max \left(0, K-s_{t}\right)\right) \\
& p_{i \rightarrow j}=\left(V \times \max \left(0, s_{t}-K\right)\right)
\end{aligned}
$$

The strike price and contracted volume components of the CfD are immutably pre-written into the smart contract. However, the smart contract does not naturally have sight of the fluctuating spot price published by the Market Operator of the pool market at every trading period. Hence, BED needs an oracle to provide external data to the smart contract [31], [32]. The oracle's role here is to simply furnish the smart contract with the spot prices of electricity traded at every period in the pool market for electricity.

After every trading period $\forall t \in T$, the escrow accounts of the contracting parties are updated based on (1) and (2). In summary, when a call is made to the function that enforces autonomous settlement, three processes are implemented sequentially. First, the spot price is obtained from the oracle. Secondly, the CfD payoff is computed within the smart contract. Lastly, the escrow accounts of the contracting parties are directly updated based on the calculated payoff. Contracting parties, as they deem fit, can then make withdrawals from their escrow account in the case of a positive CfD payoff

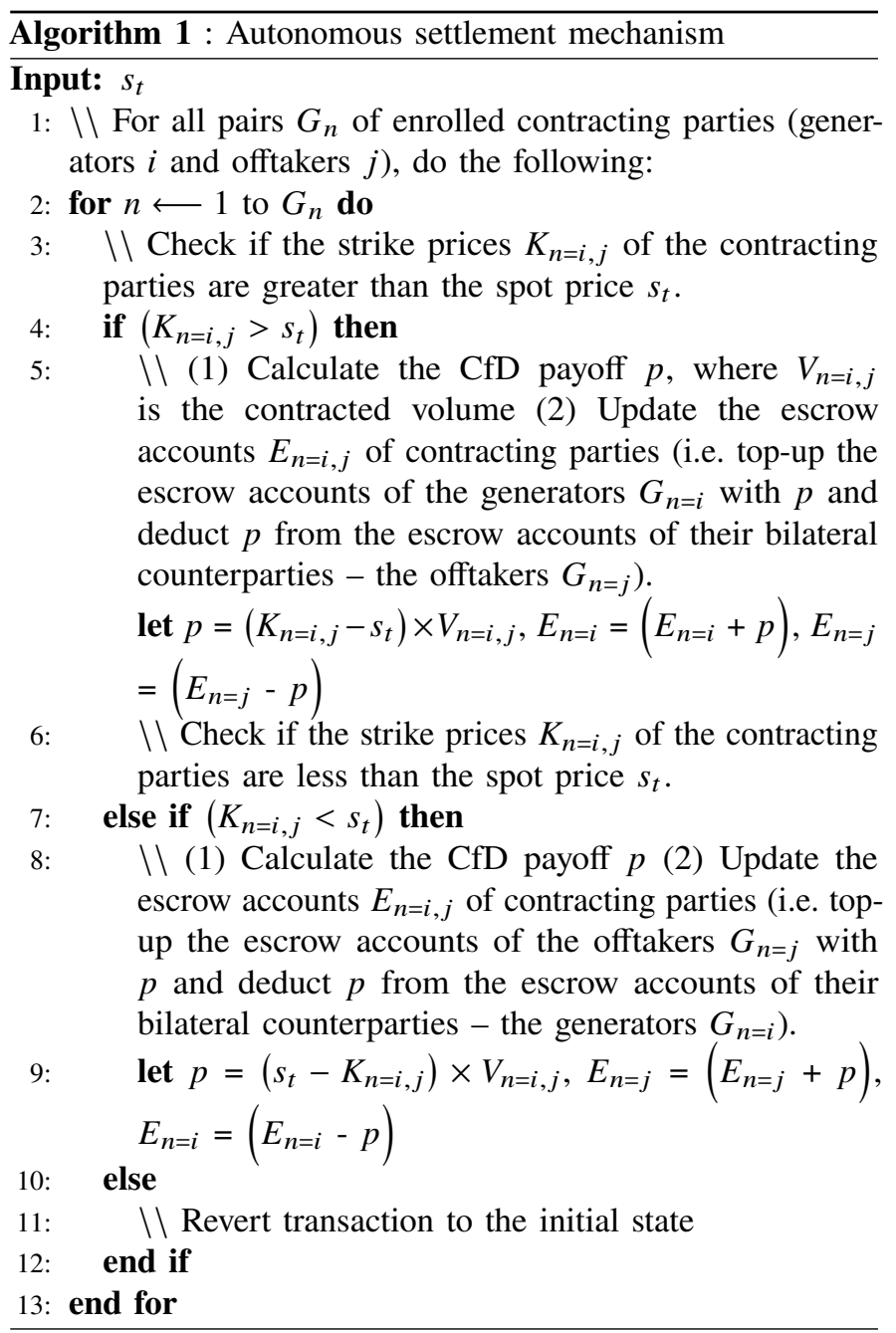

or make deposits to their escrow account in the case of a negative CfD payoff. Fig. 4 and Algorithm 1 demonstrates the sequence of execution of the autonomous settlement mechanism. Importantly, this chaincode will irrevocably and irrefutably execute once the smart contract is committed to the blockchain

\section{B. Autonomous collateralization mechanism imposed on parties enrolled in the BED smart contract}

Counterparty credit risk is one of the main risks of contracting parties in a derivative transaction because a party in a particular bilateral arrangement may not have the funds, or the willingness, to follow through on its cross-subsidizing obligations [9]. To derisk counterparty credit exposure onchain and in a fully autonomous way, we propose a novel collateralization mechanism. Here, collaterals are held within the smart contract on behalf of contracting parties for settlement purposes. This approach is unlike traditional CfDs where collaterals are held by a trusted intermediary, which exposes the contracting parties to third-party risks [29]. It is imperative that the collaterals held in escrow accounts within the smart contract are sufficient to incentivize rational behavior amongst 
contracting parties; hence, aligning with the CfD's purpose as an enduring hedging arrangement. For this reason, designing a suitable collateralization mechanism is the core problem for such a blockchain derivative instrument.

Key to collateral management is maintenance margin. The maintenance margin of a contracting party reflects its credit exposure to its counterparty in the smart contract at every instance [9]. Another important aspect of collateral is termination penalty. Termination penalties are usually included in derivative contracts that afford contracting parties the option to leave the contract before the end of the agreement duration [19]. The termination penalty must be adequate to discourage contracting parties from prematurely exiting the contract [19].

To ensure that contracting parties always have sufficient funds in their escrow accounts to make settlement payments due to their bilateral counterparty, we propose that their minimum maintenance margin $M_{\forall t \in T}^{\min }$ is equal to or greater than maximum CfD payoff $p^{\max }$ that can be due to the generator $i$ or offtaker $j$ at every trading period as in (3). This requirement is imposed in the BED smart contract because our proposed settlement mechanism ensures autonomous CfD payoffs due to contracting parties every trading period, which limits their counterparty credit exposure to only a single trading period. The maximum CfD payoff $p^{\max }$ that can be due to the generator $i$ from the offtaker $j$ at every trading period is represented in (4). On the other hand, the maximum CfD payoff $p^{\max }$ that can be due to the offtaker $j$ from the generator $i$ at every trading period is shown in (5). Therefore, the minimum maintenance margin becomes as in (6).

$$
\begin{gathered}
M_{\forall t \in T}^{\min } \geq p^{\max } \\
p_{j \longrightarrow i}^{\max }=\underset{s_{t}}{\operatorname{argmax}}\left(V \times\left(s_{t}-K\right)\right) \\
p_{i \longrightarrow j}^{\max }=\underset{s_{t}}{\operatorname{argmin}}\left(V \times\left(K-s_{t}\right)\right) \\
M_{\forall t \in T}^{\min } \geq \max \left(p_{j \longrightarrow i}^{\max }, p_{i \longrightarrow j}^{\max }\right)
\end{gathered}
$$

From (4) and (5), it is evident that the max and min limits of the volatile spot price $s_{t}$ defines the $p^{\max }$ and thus the $M_{\forall t \in T}^{\min }$ of contracting parties. Contracting parties have the liberty to propose a suitable maintenance margin during enrollment in a particular bilateral CfD arrangement in the smart contract. However, we propose that their selection must satisfy (7) to reflect the practically possible volatility band of the spot price $s_{t}$ [8]. If (7) holds, then the $M_{\forall t \in T}^{\min }$ of the BED smart contract becomes as in (8).

$$
\begin{gathered}
0 \leq s_{t} \leq 2 K \\
M_{\forall t \in T}^{\min } \geq K \times V
\end{gathered}
$$

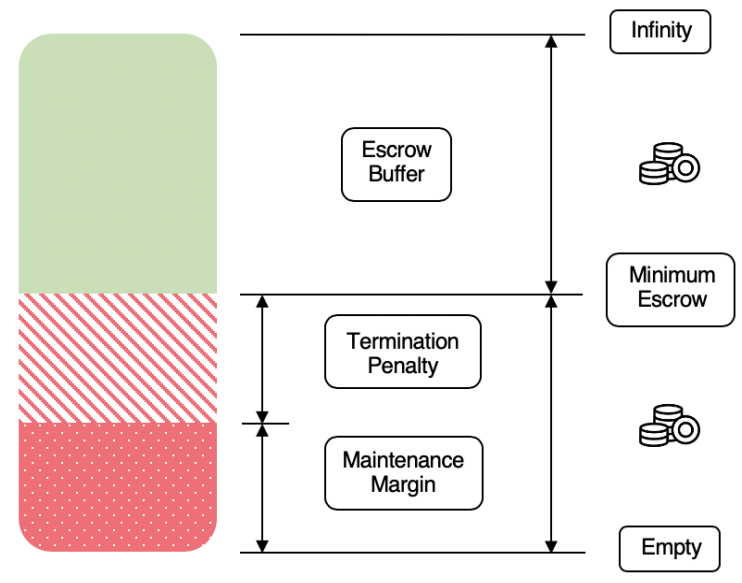

Fig. 5. Management levels of collateral to be maintained by contracting parties enrolled in the BED smart contract.

Contracting parties can exit the smart contract prematurely (i.e., before the end of the agreement duration), either voluntarily or due to a default on their minimum escrow requirement $R_{\forall t \in T}^{\min }$. However, to discourage them from exiting the smart contract prematurely, we propose a termination penalty premium payment. Contracting parties can offer a suitable termination penalty $L$ during enrollment in a particular bilateral CfD arrangement in the smart contract [1]. Therefore, the minimum escrow requirement $R_{\forall t \in T}^{\min }$ that must be maintained by each contracting party in BED is the sum of its maintenance margin $M_{\forall t \in T}^{\min }$ and termination penalty $L$ as in (9). The minimum escrow requirement essentially ensures that each contracting party has sufficient funds in their escrow account to reduce their credit risk exposure to their bilateral counterparty and to allow for termination penalty payment to their counterparty if they prematurely exit the contract.

$$
R_{\forall t \in T}^{\min }=M_{\forall t \in T}^{\min }+L
$$

Collaterals held in escrow accounts of contracting parties in the smart contract could look like Fig. 5. Contracting parties are incentivized to watch the escrow accounts of their bilateral counterparties in the smart contract and invoke a default call if they fall below the minimum escrow requirement. Hence, contracting parties are incentivized to maintain their collaterals above the minimum escrow requirement (i.e., at the escrow buffer region) to avoid liquidation of their escrow accounts in the smart contract. Nonetheless, contracting parties can choose to leave the smart contract before the end of the agreement duration in exchange for a termination penalty payment. The process for enrolling in, and prematurely exiting the smart contract are detailed in Section II-C and II-D, respectively.

\section{Enrolling in a particular bilateral CfD arrangement in the BED smart contract}

Parties that intend to enter BED must follow this four-stage route: positioning, offering, staking and registering (see Fig. 6). 


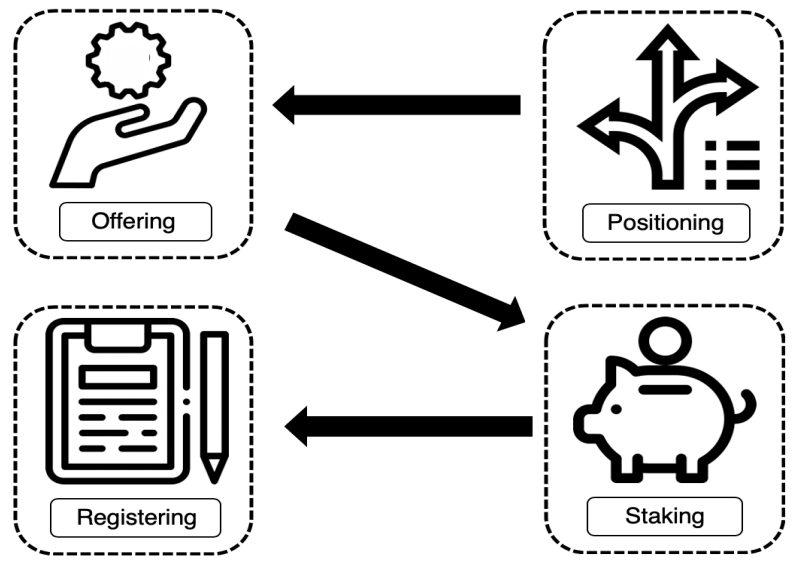

Fig. 6. Four-stage route for enrolling in a particular bilateral CfD arrangement in the BED smart contract.

First, they indicate their interest in entering the smart contract by defining their position (i.e., either as a generator or an offtaker) in the contract. While defining their position in the contract, they offer their CfD terms to the market. That is, a strike price $K$ per mwh, volume ${ }^{2} V$ in mwh, CfD agreement duration $U$ and termination penalty $L$. To discourage sham registrations, we propose that parties, while enrolling in the smart contract, place an expression of interest deposit $D$ as in (10). Essentially, the $D$ to be lodged by contracting parties must not be less than the minimum escrow requirement $R_{\forall t \in T}^{\min }$ calculation of the CfD terms they have offered. Participants can then begin observing the smart contract's events to determine when a counterparty with a similar strike price, volume, termination penalty, and agreement duration has expressed its interest to register in the contract. The ability to watch these events is usually programmable in most public blockchains and could be facilitated with a suitable front-end application [33].

$$
D \geq R_{\forall t \in T}^{\min }
$$

When a participant finds a counterparty that shares similar terms with them, they register themselves and the particular counterparty into the smart contract as a pair $G_{n}$ using the next available sequential ID pair count $\left(G_{n}+1\right)$. Alternatively, they wait until a counterparty with compatible terms registers into the smart contract as a pair. This generator-offtaker $(i, j)$ pair requirement is enforced by Algorithm 2, since BED is effectively a bilateral forward contract marketplace that requires that the number and contracting terms of generator $i$ and offtaker $j$ correspond. Following registration, the expression of interest deposit $D$ is paid into the escrow account $E_{n}$ of the bilateral contracting parties. Thereafter, they can participate in the BED

${ }^{2}$ The offered volume will typically match the quantity of electricity that is expected to be delivered in the case of a generator, or to be evacuated in the case of an offtaker, at every trading period in the pool market.

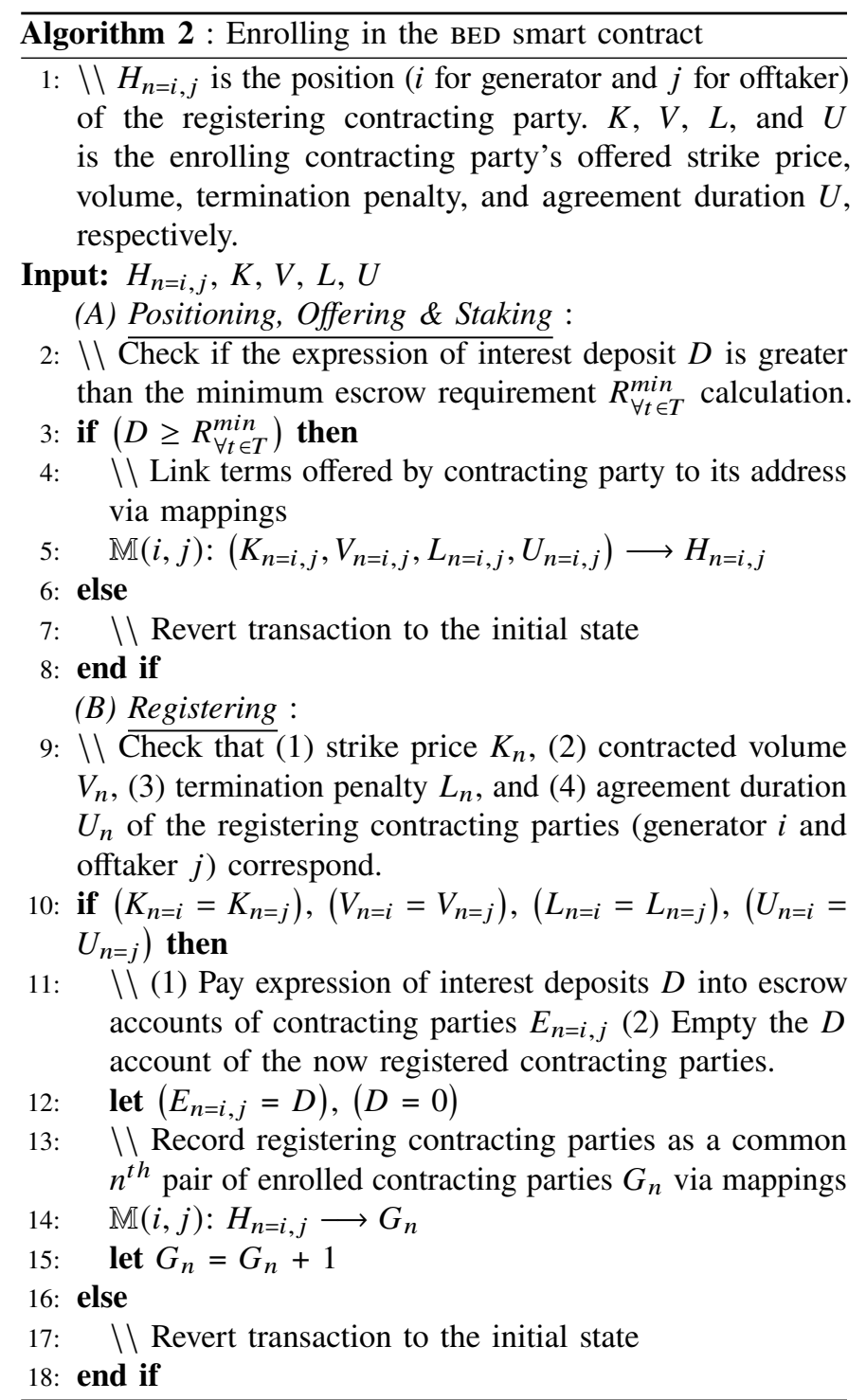

smart contract and manage their escrow accounts according to the smart contract's rules.

\section{Exiting a particular bilateral CfD arrangement in the BED smart contract prematurely}

The control flow of prematurely exiting BED, that is, leaving the BED smart contract before the end of the agreement duration, is shown in Fig. 7. Enrolled contracting parties, can prematurely exit BED either voluntarily or due to a default on their minimum escrow requirement $R_{\forall t \in T}^{\min }$. The voluntary exit option gives contracting parties the right, but not the obligation to exit the smart contract prematurely in exchange for a termination penalty $L$ payment [19]. When an enrolled contracting party calls a voluntary exit option, the termination penalty $L$ deposit of the exiting contracting party is transferred to the non-exiting contracting party. Both the exiting and non-exiting contracting 


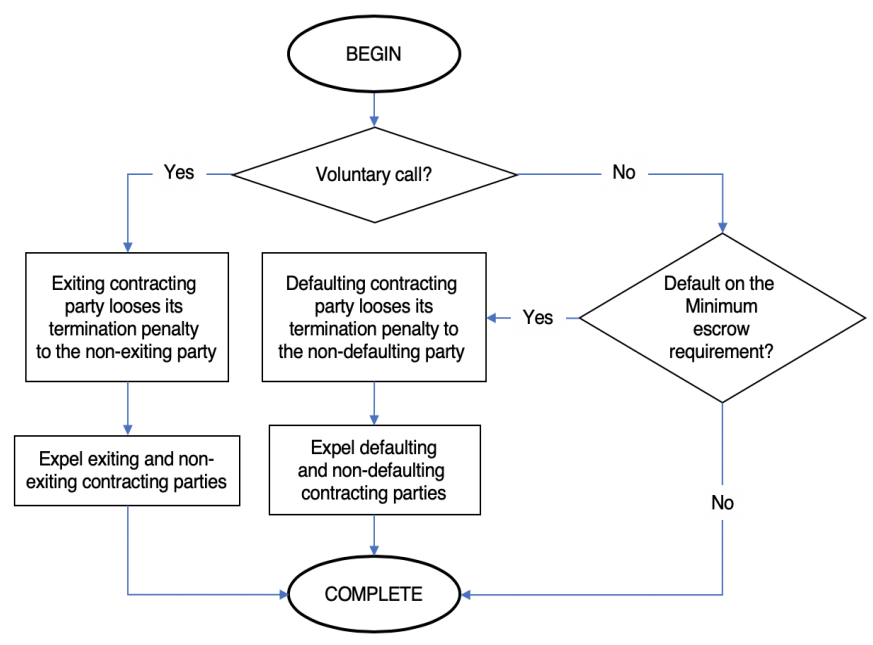

Fig. 7. Flow chart of the process for prematurely exiting a particular bilateral CfD arrangement in the BED smart contract.

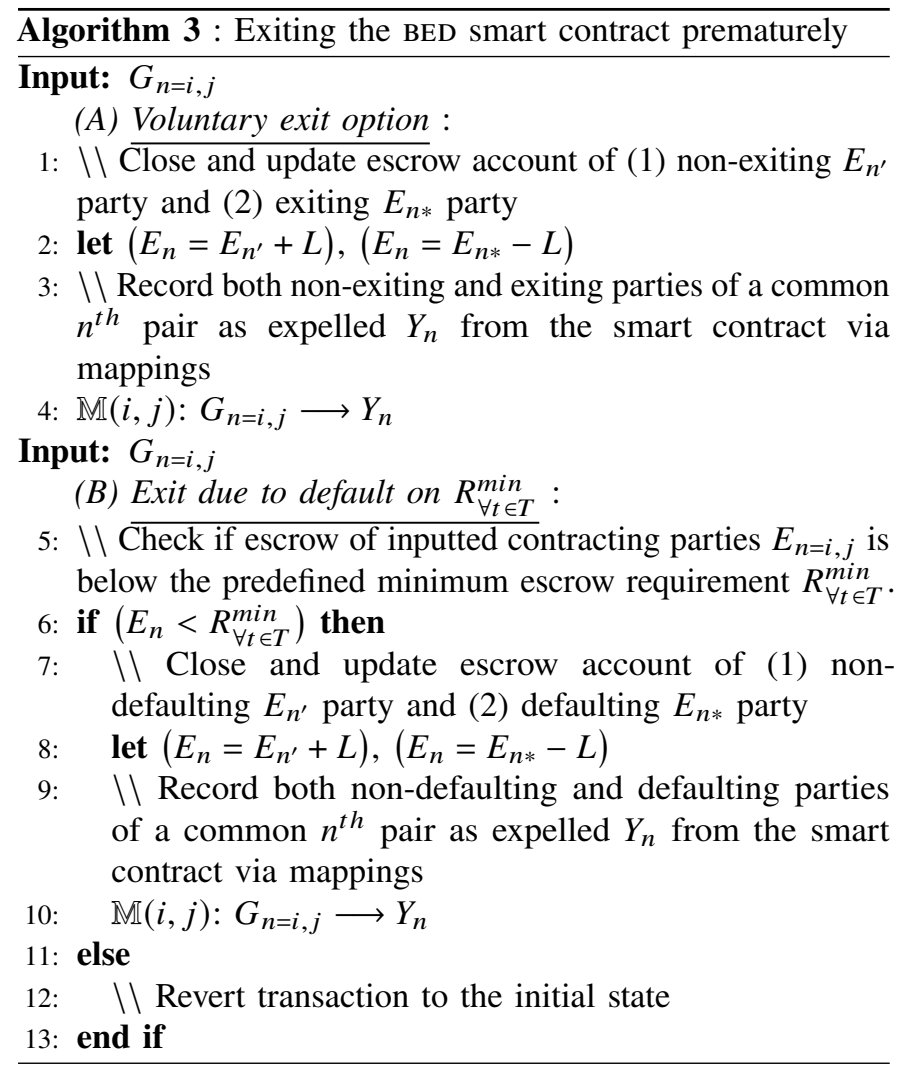

parties that share a common $n^{\text {th }}$ pair become expelled $Y_{n}$ from the smart contract (see Algorithm 3).

The alternative way contracting parties can prematurely exit the smart contract is by simply defaulting on their minimum escrow requirement. When a default call is invoked on a contracting party whose escrow account falls below the minimum escrow requirement, the defaulting and nondefaulting party that shares a common $n^{\text {th }}$ pair become expelled from the contract. Before expulsion, the termination penalty $L$ deposit of the defaulting contracting party is transferred to the non-defaulting contracting party. Contracting parties are incentivized to police the escrow accounts of their bilateral counterparty in the smart contract to ensure that they do not fall below the minimum escrow requirement. Algorithm 3 shows the process flow for contracting parties that intend to prematurely exit the smart contract.

Importantly, the smart contract becomes closed to both contracting parties that share a common $n^{\text {th }}$ pair, at the end of their bilateral CfD agreement duration. Before the smart contract is closed to the affected parties, we propose a grace period (see Fig. 8). During this time, the smart contract's operation will be limited to a single function that enables both parties to withdraw any remaining balance in their escrow account. After the grace period, parties no longer have access to the smart contract (see Fig. 8). These protocols only apply to parties who have come to the end of their bilateral CfD agreement. Other contracting parties remain in the BED smart contract and continue to participate according to the smart contract's rules until the end of their respective CfD agreement duration.

\section{Test Platform}

This section describes the test platform employed to demonstrate the functioning and value of the BED financial instrument.

\section{A. Selected parameters for the autonomous collateralization and settlement mechanisms}

To showcase our novel autonomous collateralization and settlement mechanisms, we assume that three renewable generators have separately enrolled in BED to hedge against their trading period spot price risk in the day-ahead electricity market with three offtakers (see Table I). The renewable generators have a diversified portfolio of technologies that include large-scale wind, concentrated solar, and solar PV plants. Therefore, they can consistently deliver huge electricity volumes in the pool market over a 24-hour period. The generators have a common agreement duration of 2 years with their bilateral counterparty - an offtaker. Their agreement is also Us\$-denominated and includes a pre-agreed strike price, contracted volume, and termination penalty with the same bilateral counterparty (see Table II). Lastly, their minimum maintenance margin is determined as per Equation (8), i.e., $\left(M_{\forall t \in T}^{\min } \geq K \times V\right)$.

\section{B. Availability of blockchain tokens pegged to fiat currencies}

For demonstration purposes, we assume that a collateralbacked stablecoin has been integrated into our smart contract. Collateral-backed stablecoins achieve their pegging with fiat via overcollaterization of a basket of cryptoassets locked in smart contract accounts [28]. They maintain a 1:1 pegging with fiat currencies, the currencies that the clearing prices of the 


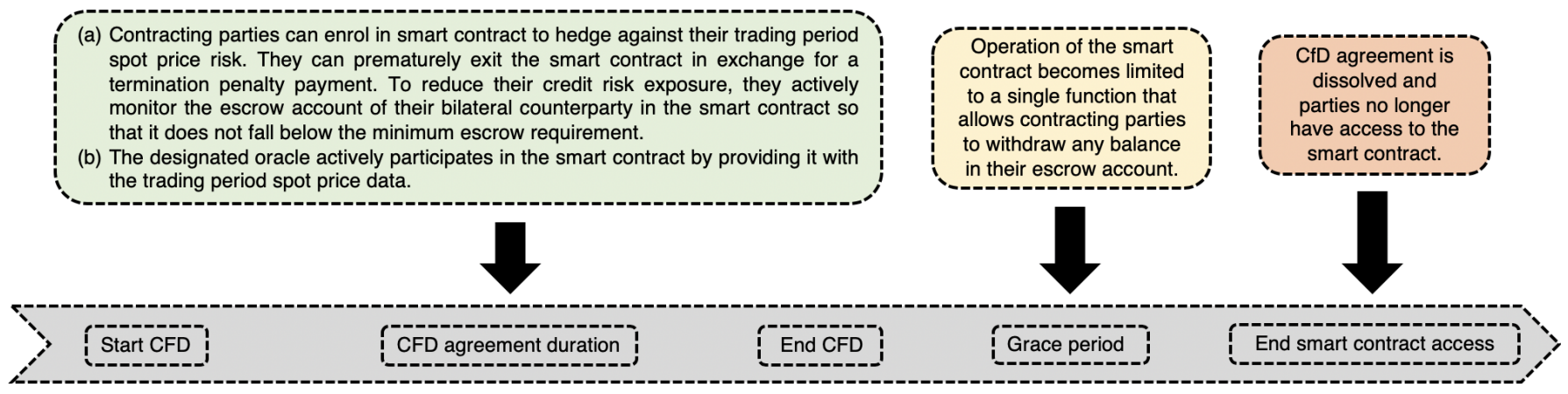

Fig. 8. Simplified timelines of contracting parties participating in a particular bilateral CfD agreement in the BED smart contract.

TABLE I

SPOT PRICES ON THREE DIFFERENT AND NON-SEQUENTIAL TRADING DAYS

\begin{tabular}{lccc}
\hline $\begin{array}{l}\text { Trading } \\
\text { periods }\end{array}$ & $\begin{array}{c}\text { Day } \mathbf{1} \\
\text { (US\$/MWh) }\end{array}$ & $\begin{array}{c}\text { Day 2 } \\
\text { (US\$/MWh) }\end{array}$ & $\begin{array}{c}\text { Day } \mathbf{3} \\
\text { (US\$/MWh) }\end{array}$ \\
\hline $1(00: 00)$ & 85 & 82 & 87 \\
$2(01: 00)$ & 76 & 72 & 71 \\
$3(02: 00)$ & 81 & 80 & 80 \\
$4(03: 00)$ & 73 & 80 & 75 \\
$5(04: 00)$ & 73 & 72 & 75 \\
$6(05: 00)$ & 72 & 83 & 76 \\
$7(06: 00)$ & 86 & 74 & 75 \\
$8(07: 00)$ & 76 & 76 & 79 \\
$9(08: 00)$ & 87 & 86 & 86 \\
$10(09: 00)$ & 76 & 76 & 74 \\
$11(10: 00)$ & 82 & 76 & 80 \\
$12(11: 00)$ & 82 & 72 & 85 \\
$13(12: 00)$ & 83 & 89 & 73 \\
$14(13: 00)$ & 76 & 75 & 88 \\
$15(14: 00)$ & 89 & 82 & 77 \\
$16(15: 00)$ & 75 & 88 & 81 \\
$17(16: 00)$ & 86 & 83 & 87 \\
$18(17: 00)$ & 84 & 72 & 73 \\
$19(18: 00)$ & 77 & 84 & 84 \\
$20(19: 00)$ & 82 & 75 & 88 \\
$21(20: 00)$ & 71 & 86 & 72 \\
$22(21: 00)$ & 77 & 88 & 78 \\
$23(22: 00)$ & 75 & 82 & 74 \\
$24(23: 00)$ & 84 & 76 & 89 \\
\hline
\end{tabular}

TABLE II

CFD AGREEMENTS BETWEEN THREE PAIRS OF CONTRACTING PARTIES

\begin{tabular}{ccccc}
\hline Pair & $\begin{array}{c}\text { Strike } \\
\text { price } \\
\text { (US\$/MWh) }\end{array}$ & $\begin{array}{c}\text { Contracted } \\
\text { volume } \\
\text { (MWh) }\end{array}$ & $\begin{array}{c}\text { Minimum } \\
\text { maintenance } \\
\text { margin (us\$) }\end{array}$ & $\begin{array}{c}\text { Termination } \\
\text { penalty (us\$) }\end{array}$ \\
\hline 1 & 78 & 650 & 50,700 & 76,050 \\
2 & 74 & 700 & 51,800 & 62,160 \\
3 & 82 & 680 & 55,760 & 100,368 \\
\hline
\end{tabular}

pool market for electricity are usually denominated in. Hence, we maintain that 1 stablecoin is equals us $\$ 1$. The stablecoin service introduces an attack vector and thus exposes the smart contract to security risks. We note that stablecoin services [28] are a mature technology that presently underpins several DeFi applications, such as in [30].

\section{Availability of trustworthy oracle service providing clearing prices of the pool market for electricity}

For demonstration purposes, we assume that a decentralized oracle service such as in [30], serves as a trustworthy oracle in BED. While the smart contract can read and react to the data fed by the decentralized oracle service autonomously, a malicious party could manipulate this data stream to game the operation of the smart contract [31], [32]. Hence, the oracle service introduces a possible attack vector and thus exposes the smart contract to security risks. Again, we consider this broader issue to lie beyond the scope of the present work.

\section{Results And Discussion}

\section{A. Results}

To illustrate the operation of our novel autonomous settlement and collateralization mechanisms, and their usefulness; we examine the escrow accounts of the three separate pairs of participants enrolled in the BED smart contract. We analyze their escrow accounts on three different and non-sequential trading days as in Table I. That is, pair $G_{1}$ on trading day 1 , $G_{2}$ on trading day 2 and pair $G_{3}$ on trading day 3 (see Figs. 9,10 and 11). In all trading days, both contracting parties of the common pair commence their respective trading day with the same collateral balance in their escrow accounts.

In the case of $G_{1}$ (i.e., at trading day 1 ), both contracting parties remain in the smart contract over the trading day and abide by the escrow management requirements of BED. For $G_{2}$ (i.e., at trading day 2), both contracting parties conform to the escrow management requirements of BED, until trading period 21 (20:00) when the offtaker decides to exit the contract. Its counterparty - the renewable generator - also becomes immediately expelled from the contract. Before being expelled, it receives a termination penalty compensation of us $\$ 62,160$ from the offtaker. For $G_{3}$ (i.e at trading day 3), both contracting parties observe the escrow management requirements of the smart contract until trading period 22 (21:00), when the offtaker defaults on the minimum escrow requirement of the contract. A default call is immediately invoked by its counterparty. The escrow accounts of both contracting parties get liquidated from 


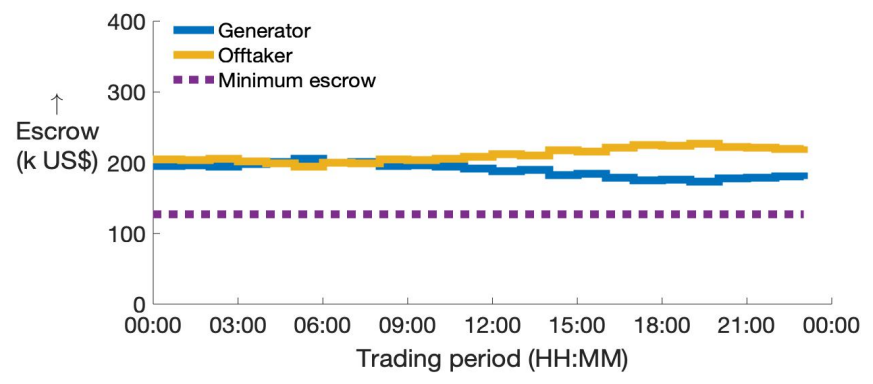

Fig. 9. PAIR 1: Escrow accounts of pair of participants on trading day 1.

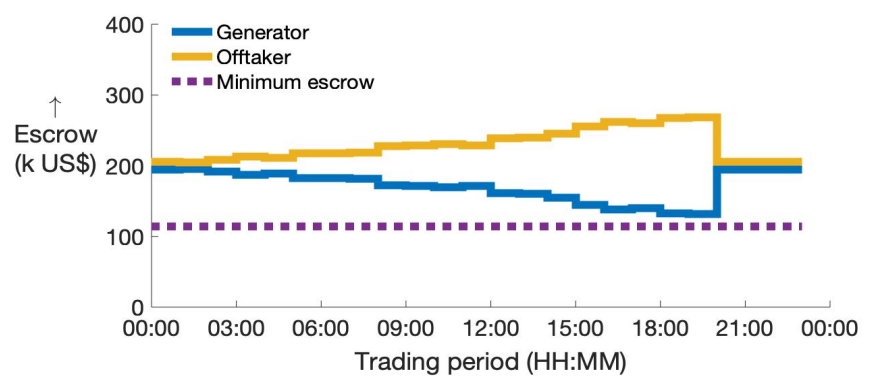

Fig. 10. PAIR 2: Escrow accounts of pair of participants on trading day 2.

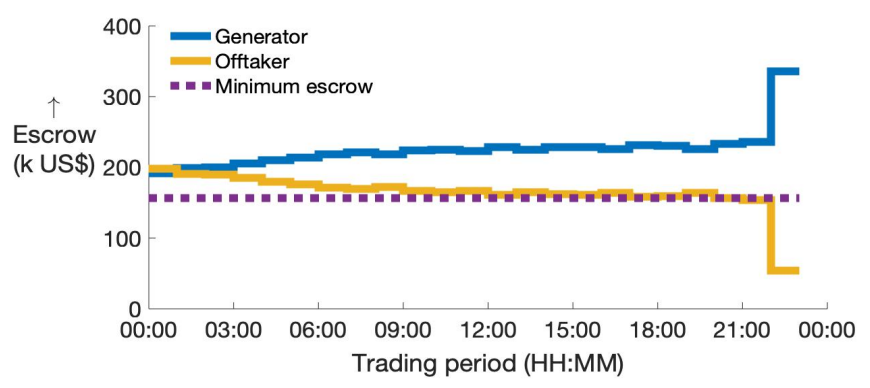

Fig. 11. PAIR 3: Escrow accounts of pair of participants on trading day 3.

the smart contract. Like in the case of $G_{2}$, the defaulting party - the offtaker - forfeits its termination penalty deposit of US\$ 100,368 to the generator.

To show the impact of the rapid settlement times of our approach on contracting parties, we examine the net cash flows of the renewable generators. Suppose we assume that unlike Figs. 9, 10 and 11; the generators maintain their escrow accounts at an $80 \%$ level (i.e., they deposit $80 \%$ to their escrow account following a negative payoff and withdraw $80 \%$ from their escrow account following a positive payoff at every trading period), then their net cash flows become as in Figs. 12, 13 and 14 respectively. From Figs. 12, 13 and 14, it is evident that the hourly net cash flows of the generators enrolled in BED are flatter than that of the traditional CfD. Essentially, traditional CfD instruments are unable to have any effect on the hourly or daily cash flows of contracting parties participating in the day-ahead electricity market because payoffs in traditional CfD instruments occur after many days at least [10]. It is important

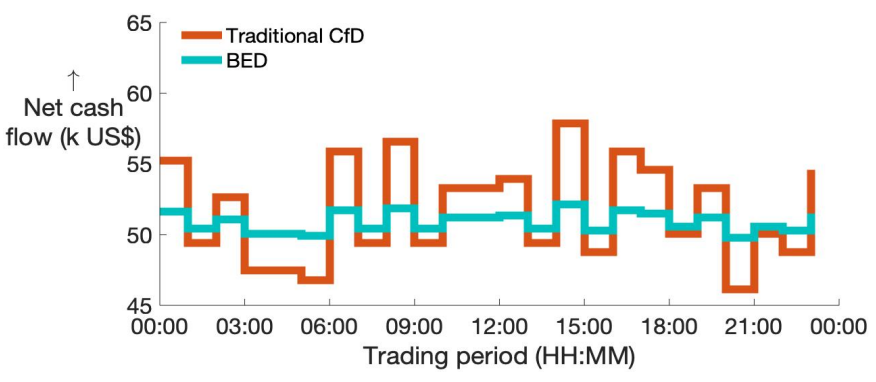

Fig. 12. PAIR 1: Net cash flow of generator on trading day 1.

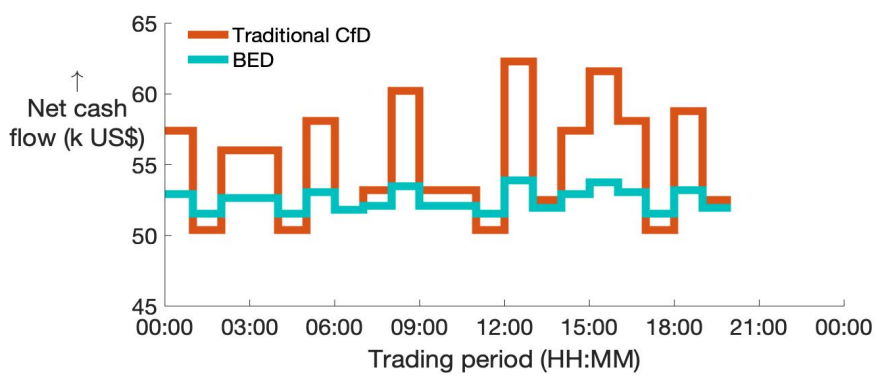

Fig. 13. PAIR 2: Net cash flow of generator on trading day 2 .

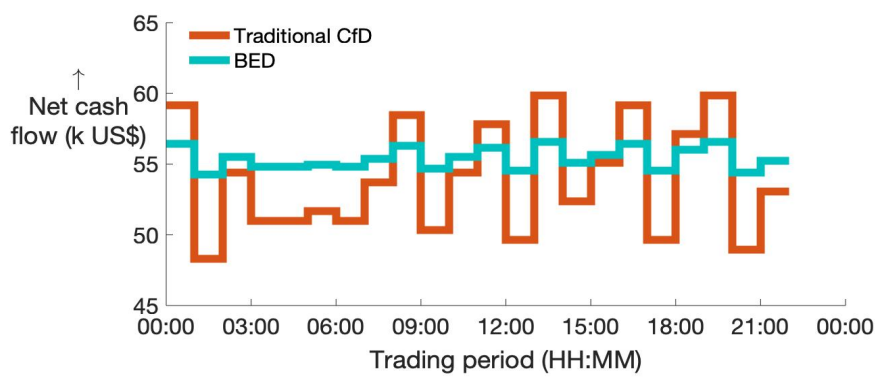

Fig. 14. PAIR 3: Net cash flow of generator on trading day 3.

to note that although BED settles more frequently (lowering margining risk) compared to traditional CfD instruments, the net cash flow of traditional CfD instruments after settlement is the same as that of BED.

\section{B. Discussion}

Counterparty credit risk is one of the main risks of contracting parties in a derivative transaction [29]. It is also the hardest risk to hedge since it is innately part of every CfD transaction. As already discussed, in traditional CfD instruments, collateral held by an intermediary could be used to hedge against this risk exposure. However, from the results in Section IV-A, it is evident that counterparty credit risk can be mitigated onchain and without the need for an intermediary, known to expose the contracting parties to third-party risks. In traditional CfD instruments, collaterals are also usually large, given that clearing and settlement take many days at least, or even up to months in some jurisdiction [10]. These large collateral 
requirements introduce a new risk, known as margining risk [9]. From the results in Section IV-A, this type of risk is low in BED because collateral requirements are low, resulting from the fact that payoffs are settled as the market clears hourly.

The risks introduced by intermediaries in over-the-counter contracts are also significant. For instance, in traditional overthe-counter contracts, both contracting parties rely on an intermediary to handle and manage their collaterals [9]. What then happens if this entity is fraudulent, unreliable, or becomes insolvent? In BED, the smart contract acts as the intermediary - safely holding the collaterals of contracting parties in the contract. Moreover, smart contracts are dependable because they are persisting and unmistakably function as per the predefined conditions embedded in them [1]. The decentralized network of blockchain also makes smart contract transactions transparent and hence reduces the probability of fraud.

Further, unlike traditional over-the-counter contracts, participating in BED is cost-effective. The automation of processes essentially removes some of the cost components incurred in traditional CfD instruments. These costs are usually associated with clearing \& settlement, enforcement \& compliance, adjudication processes, etc. [5]. The market-based feature of BED should also make it more liquid and cost-effective than traditional CfD instruments.

The BED community is not governed by a government regulatory body or federal court. BED effectively acts as the government, regulator, and judge - punishing negligent parties and rewarding active and contributing parties. Moreover, contracting parties in BED do not have to trust or know each other to act fairly. Rather, the smart governance system enforces good behavior through interconnected financial incentives.

To say BED is risk-free will be untrue. First, building a secure smart contract currently does not imply that it is invulnerable to attackers. This is because smart contracts on the blockchain could be susceptible to novel attack paths [34]. The stablecoin [28] and oracle [31], [32] services could also expose the smart contract to security risks. BED is susceptible to account risks related to the possible loss of private keys and unintended funds transfer to the wrong address [4]. The account risks are significant because in blockchain networks, cryptocurrency ownership is held in digital keys, and if they are lost, funds associated with them become irrecoverable. In the same vein, unintended fund transfer to a wrong address implies a permanent loss of funds [4]. Since the blockchain ecosystem is still burgeoning, some of these risks could likely be mitigated or outrightly eliminated soon.

\section{Conclusion}

In this paper, we have built upon traditional CfD instruments to demonstrate the plausibility of blockchain applications in electricity derivatives. From the results, it is evident that a blockchain-based CfD may outperform a traditional CfD in certain respect. As this research represents a novel DeFi derivative instrument in the electricity industry, future research
TABLE III

RISK COMPARISON BETWEEN BED AND TRADITIONAL CFD INSTRUMENTS

\begin{tabular}{lcc}
\hline Risk & Traditional CfD & BED \\
\hline Credit & Moderate & Low \\
Margining & High & Low \\
Third-party & High & Low \\
Legal & High & Low \\
Process & High & Low \\
Liquidity & High & Moderate \\
Account & Low & High \\
Volatility & Low & Low \\
Design & Low & Low \\
Security & Low & High \\
\hline
\end{tabular}

can leverage key features of our business logic to test the feasibility of other types of electricity derivatives on the blockchain.

\section{REFERENCES}

[1] O. Alao and P. Cuffe, "Towards a Blockchain Contractfor-Difference Financial Instrument for Hedging Renewable Electricity Transactions," in IEEE TechRxiv, 2020, pp. 1-6.

[2] Min Liu, F. F. Wu, and Yixin Ni, "A survey on risk management in electricity markets," in 2006 IEEE Power Engineering Society General Meeting, 2006.

[3] S. Bhattacharya, A. Gupta, K. Kar, and A. Owusu, "Hedging strategies for risk reduction through weather derivatives in renewable energy markets," in 2015 IEEE International Conference on Renewable Energy Research and Applications (ICRERA), 2015, pp. 1190-1195.

[4] O. Alao and P. Cuffe, "Towards a Blockchain Special Purpose Vehicle for Financing Independent Renewable Electricity Projects in Sub-Saharan Africa," in IEEE TechRxiv, 2020, pp. 1-6.

[5] P. Simshauser, "On the Stability of Energy-Only Markets with Government-Initiated Contracts-for-Differences," Energies, vol. 12, no. 13, p. 2566, 2019.

[6] S. Pineda and A. J. Conejo, "Using electricity options to hedge against financial risks of power producers," IEEE Journal of Modern Power Systems and Clean Energy, vol. 1, no. 2, pp. 101-109, 2013.

[7] S. J. Deng and S. S. Oren, "Electricity derivatives and risk management," Energy, vol. 31, no. 6-7, pp. 940-953, 2006, ISSN: 03605442. DOI: 10.1016/j.energy.2005.02.015.

[8] ENTSOE, Day-ahead Prices, 2020. [Online]. Available: https: //transparency.entsoe.eu.

[9] J. Hull, Options, Futures and Other Derivatives. 2009, ISBN: 0135009944. DOI: 10.1007/978-1-4419-9230-7_2.

[10] ESB, "Master Contract for Difference Agreement Between Electricity Supply Board and [Buyer] Being a PSO-Supported Contract Issued on [Date]," Tech. Rep., 2013.

[11] Y. Chen and C. Bellavitis, "Blockchain Disruption and Decentralized Finance: The Rise of Decentralized Business Models," Journal of Business Venturing Insights, vol. 13, 2020.

[12] M. F. Zia, M. Benbouzid, E. Elbouchikhi, S. M. Muyeen, K. Techato, and J. M. Guerrero, "Microgrid Transactive Energy: Review, Architectures, Distributed Ledger Technologies, and Market Analysis," IEEE Access, vol. 8, pp. 19410-19432, 2020, ISSN: 2169-3536. DOI: 10.1109/ACCESS.2020.2968402. 
[13] M. T. Devine and P. Cuffe, "Blockchain electricity trading under demurrage," IEEE Transactions on Smart Grid, vol. 10, no. 2, pp. 2323-2325, 2019, ISSN: 1949-3053. DOI: 10.1109/ TSG.2019.2892554.

[14] S. Wang, L. Ouyang, Y. Yuan, X. Ni, X. Han, and F. Wang, "Blockchain-enabled smart contracts: Architecture, applications, and future trends," IEEE Transactions on Systems, Man, and Cybernetics: Systems, vol. 49, no. 11, pp. 2266-2277, 2019.

[15] K. Christidis and M. Devetsikiotis, "Blockchains and Smart Contracts for the Internet of Things," IEEE Access, vol. 4, pp. 2292-2303, 2016, ISSN: 21693536. DOI: 10.1109/ACCESS. 2016.2566339.

[16] C. I. Dick and A. Praktiknjo, "Blockchain technology and electricity wholesale markets: Expert insights on potentials and challenges for OTC trading in Europe," Energies, vol. 12, no. 5, 2019, ISSN: 19961073. DOI: 10.3390/en12050832.

[17] O. Jayeola, "Inefficiencies in trade reporting for over-thecounter derivatives: Is blockchain the solution?" Capital Markets Law Journal, vol. 15, no. 1, pp. 48-69, 2020, ISSN: 17507227. DOI: $10.1093 / \mathrm{cmlj} / \mathrm{kmz} 028$.

[18] M. Morini, "Managing Derivatives on a Blockchain. A Financial Market Professional Implementation," SSRN Electronic Journal, 2017. [Online]. Available: https://ssrn.com/abstract= 3075540.

[19] C. P. Fries and P. Kohl-landgraf, "Smart Derivative Contracts: Detaching Transactions from Counterparty Credit Risk," pp. 122, 2018.

[20] Coin Telegraph, Derivatives in Crypto, Explained, 2019. [Online]. Available: https://cointelegraph.com/explained/ derivatives-in-crypto-explained.

[21] M. Ul Hassan, M. H. Rehmani, and J. Chen, "DEAL: Differentially Private Auction for Blockchain based Microgrids Energy Trading," IEEE Transactions on Services Computing, vol. PP, no. c, pp. 1-1, 2019. DoI: 10.1109/tsc.2019.2947471.

[22] A. Jindal, G. S. S. Aujla, N. Kumar, and M. Villari, "GUARDIAN: Blockchain-based Secure Demand Response Management in Smart Grid System," IEEE Transactions on Services Computing, pp. 1-1, 2019, ISSN: 2372-0204. DOI: 10.1109/TSC.2019.2962677.

[23] S. Wang, A. F. Taha, J. Wang, K. Kvaternik, and A. Hahn, "Energy crowdsourcing and peer-to-peer energy trading in blockchain-enabled smart grids," IEEE Transactions on Systems, Man, and Cybernetics: Systems, vol. 49, no. 8, pp. 1612 1623, 2019, ISSN: 2168-2232. DOI: 10.1109/TSMC.2019. 2916565.

[24] A. de Villiers and P. Cuffe, "A three-tier framework for understanding disruption trajectories for blockchain in the electricity industry," IEEE Access, vol. 8, pp. 65670-65 682, 2020.

[25] Y. Huang, Y. Bian, R. Li, J. L. Zhao, and P. Shi, "Smart contract security: A software lifecycle perspective," IEEE Access, vol. 7, pp. $150184-150202,2019$.

[26] C. G. Harris, "The risks and challenges of implementing ethereum smart contracts," in 2019 IEEE International Conference on Blockchain and Cryptocurrency (ICBC), 2019, pp. 104-107.

[27] X. Wang, J. He, Z. Xie, G. Zhao, and S. C. Cheung, "ContractGuard: Defend Ethereum Smart Contracts with Embedded Intrusion Detection," IEEE Transactions on Services Computing, pp. 1-1, 2019, ISSN: 2372-0204. DOI: 10.1109/ TSC.2019.2949561.

[28] Maker Team, “The Dai Stablecoin System,” Tech. Rep., 2017.

[29] A. Asgaonkar and B. Krishnamachari, "Solving the Buyer and Seller's Dilemma : A Dual-Deposit Escrow Smart Contract for Provably Cheat-Proof Delivery and Payment for a Digital
Good without a Trusted Mediator," pp. 1-7, 2018. arXiv arXiv: $1806.08379 \mathrm{v} 1$

[30] J. Peterson, J. Krug, M. Zoltu, A. K. Williams, and S. Alexander, "Augur: a decentralized oracle and prediction market platform," pp. 1-16, 2015. DOI: 10.13140/2.1.1431.4563. arXiv: 1501.01042 .

[31] J. Adler and et al., "ASTRAEA: A Decentralized Blockchain Oracle," in 2018 IEEE International Conference on iThings, GreenCom, CPSCom and SmartData, 2018, pp. 1349-1354, ISBN: 9781538679753. DOI: 10.1109/Cybermatics.

[32] M. Merlini, N. Veira, R. Berryhill, and A. Veneris, "On Public Decentralized Ledger Oracles via a Paired-Question Protocol," ICBC 2019 - IEEE International Conference on Blockchain and Cryptocurrency, pp. 337-344, 2019. DOI: 10.1109/BLOC. 2019.8751484.

[33] A. M. Antonopoulos and G. Wood, Mastering Ethereum. 2018, ISBN: 9783540773405

[34] S. Rouhani and R. Deters, "Security, performance, and applications of smart contracts: A systematic survey," IEEE Access, vol. 7, pp. 50759-50779, 2019, ISSN: 21693536. DOI: 10.1109/ACCESS.2019.2911031. 